\title{
Iterative proportional scaling via decomposable submodels for contingency tables
}

\author{
Yushi Endo and Akimichi Takemura \\ Graduate School of Information Science and Technology \\ University of Tokyo
}

November, 2008

\begin{abstract}
We propose iterative proportional scaling (IPS) via decomposable submodels for maximizing likelihood function of a hierarchical model for contingency tables. In ordinary IPS the proportional scaling is performed by cycling through the members of the generating class of a hierarchical model. We propose to adjust more marginals at each step. This is accomplished by expressing the generating class as a union of decomposable submodels and cycling through the decomposable models. We prove convergence of our proposed procedure, if the amount of scaling is adjusted properly at each step. We also analyze the proposed algorithms around the maximum likelihood estimate (MLE) in detail. Faster convergence of our proposed procedure is illustrated by numerical examples.
\end{abstract}

Keywords and phrases: decomposable model, hierarchical model, I-projection, iterative proportional fitting, Kullback-Leibler divergence.

\section{Introduction}

Iterative proportional scaling algorithm for contingency tables, first proposed by Deming and Stephan [8], has been well studied and generalized by many authors. Ireland and Kullback [11] proved convergence of IPS and Fienberg [10] gave a simpler proof of convergence from geometric consideration. Darroch and Ratcliff [6] made a generalization to IPS and its geometrical property was studied by Csiszár [5]. Csiszár [4] also gave a more general proof of convergence and justified IPS in a general framework. Extension of IPS to continuous case was studied in Kullback [14] and Rüschendorf [17]. Effective algorithms and implementations of IPS have been also studied by many authors, including [1, [9], [12], [13], [16].

In this paper, we propose another generalization of IPS based on decomposable submodels. Decomposable models or graph decompositions have been already considered 
by Jiroušek [12, Jiroušek and Přeučil [13] and Malvestuto [16]. However they used decomposable models for efficient implementation of conventional IPS in the form of treecomputation. Here we use decomposable submodels for generalizing IPS itself. In our algorithm we adjust a larger set of marginals than the conventional IPS. The set of marginals form the generating class of a decomposable submodel. By adjusting more marginals, our proposed algorithm achieves a faster convergence to the maximum likelihood estimate than the conventional IPS, although at present it seems difficult to theoretically prove that our procedure is always faster. We prove convergence of our proposed procedure, if we adjust the amount of scaling at each step. We also analyze in detail the behavior of the proposed algorithms around the maximum likelihood estimate. As shown in Section 4 our procedure works well in practice without adjusting the amount of scaling at each step.

As suggested by a referee, it is an important topic to combine the idea of the present paper and the tree-computation approach for efficient implementation of IPS. Although we do not give a general result, in Section 4 we investigate the combination in the case of cycle models and show effectiveness of the combination by numerical experiments.

The organization of this paper is as follows. In Section 2 we summarize notations and basic facts on hierarchical models and decomposable models for multiway contingency tables. In Section 3 we propose a generalized IPS via decomposable submodels, prove its convergence and clarify its behavior close to the maximum likelihood estimate. In Section 4 we perform some numerical experiments to illustrate the effectiveness of the proposed procedure. Some discussions are given in Section 5 .

\section{Preliminaries}

In this section we summarize notations and preliminary materials on decomposable models and conventional IPS.

We follow the notation of Lauritzen [15]. Let $\Delta$ denote the set of variables of a multiway contingency table. For each $\delta \in \Delta, \mathcal{I}_{\delta}=\left\{1,2, \ldots, I_{\delta}\right\}$ denotes the set of levels of $\delta$. The set of cells is denoted by $\mathcal{I}=\times_{\delta \in \Delta} \mathcal{I}_{\delta}$. Let $n(i)$ denote the frequency of a cell $i \in \mathcal{I}$ and let $n=\sum_{i \in \mathcal{I}} n(i)$ denote the total sample size. Throughout the paper we denote the relative frequency (empirical distribution) by $r(i)=n(i) / n$. For a cell $i$ and a subset of variables $a \subset \Delta$, the marginal cell of $i$ for $a$ is denoted by $i_{a} \in \mathcal{I}_{a}=\times_{\delta \in a} \mathcal{I}_{\delta}$, the marginal of $r$ on $a$ is denoted by $r[a]$, and the marginal relative frequency of $a$ is denoted by $r\left(i_{a}\right)$.

The generating class of a hierarchical model is the family of the variable sets indexing the maximal interaction terms in the hierarchical model. We denote a hierarchical model with generating class $\mathcal{C}$ by $M(\mathcal{C})$, and call the sets in $\mathcal{C}$ the generators of $M(\mathcal{C})$. A hierarchical model is a decomposable model if there exists an ordering $\left(C_{1}, \ldots, C_{m}\right)$ of its generators that satisfies the running intersection property:

(RIP) For each $j(2 \leq j \leq m)$, there exists $k(1 \leq k \leq j-1)$, such that

$$
C_{j} \cap\left(C_{1} \cup C_{2} \cup \cdots \cup C_{j-1}\right) \subset C_{k} .
$$


Such an ordering is called a perfect sequence. Given a perfect sequence $\left(C_{1}, \ldots, C_{m}\right)$ of the generators of a decomposable model, let

$$
S_{j}=C_{j} \cap\left(C_{1} \cup C_{2} \cup \cdots \cup C_{j-1}\right) . \quad(2 \leq j \leq m)
$$

If no $S_{j}$ is the empty set, then the decomposable model is said to be connected. If this is the case, then each set $S_{j}$ is called a separator of the generating class of the decomposable model; moreover, both the generators of the decomposable model and the separators of its generating class can be graphically viewed as being the (maximal) cliques and the minimal vertex separators of a suitable chordal, connected graph, sometimes called the "adjacency graph" of the generating class of the decomposable model. In what follows, we always assume that a decomposable model is connected. In this paper

$$
\mathcal{S}=\left\{S_{2}, \ldots, S_{m}\right\}
$$

denotes the multiset of separators. The number of times a separator $S$ appears in $\mathcal{S}$ is called the multiplicity of $S$.

The MLE of the cell probabilities $\{p(i)\}$ under a hierarchical model $M(\mathcal{C})$ is given by the probability distribution denoted by $p_{\mathcal{C}}$ that belongs to $M(\mathcal{C})$ and satisfies the marginality constraints

$$
p_{\mathcal{C}}[C]=r[C], \quad \forall C \in \mathcal{C} .
$$

Equivalently, $p_{\mathcal{C}}$ is the extension of the set of probability distributions $\{r[C]: C \in \mathcal{C}\}$ that has the maximum entropy. If $M(\mathcal{C})$ is a decomposable model then $p_{\mathcal{C}}(i)$ has the following product-form expression:

$$
p_{\mathcal{C}}(i)= \begin{cases}\frac{\prod_{C \in \mathcal{C}} r\left(i_{C}\right)}{\prod_{S \in \mathcal{S}} r\left(i_{S}\right)}, & \text { if } r\left(i_{C}\right)>0, \forall C \in \mathcal{C}, \\ 0, & \text { otherwise }\end{cases}
$$

In the following we call $p_{\mathcal{C}}$ in (2) the maximum-entropy extension of the set of probability distributions $\{r[C]: C \in \mathcal{C}\}$. In Algorithm 2 below, we use the maximum-entropy extension of the form (21) of the set $\{q[C]: C \in \mathcal{C}\}$ even when $q$ is not necessarily normalized to be a probability distribution.

For obtaining MLE for other graphical or hierarchical models we need some iterative procedure. The following conventional IPS, cycling through the elements of the generating class, is commonly used for this purpose. In the following let $p^{(t)}(i)$ denote the estimate of the probability of the cell $i$ at the $t$-th step of iteration and let $p^{(t)}=\left\{p^{(t)}(i)\right\}$.

\section{Algorithm 0 (Conventional IPS)}

Let $p^{(0)}(i) \equiv 1 /|\mathcal{I}|$. The updating formula is given as

$$
p^{(t+1)}=p^{(t)} \times \frac{r[C]}{p^{(t)}[C]},
$$

where $C=C_{j}, \quad j=(t \bmod m)+1$. 
The Kullback-Leibler divergence (KL-divergence) from a probability distribution $p$ to another probability distribution $q$ is denoted by

$$
I(p: q)=\sum_{i \in \mathcal{I}} p(i) \log \frac{p(i)}{q(i)} .
$$

The $\log$ sum inequality (Chapter 2 of [3]) for non-negative numbers $a_{1}, \ldots, a_{N}$ and $b_{1}, \ldots, b_{N}$ is

$$
\sum_{i=1}^{N} a_{i} \log \frac{a_{i}}{b_{i}} \geq a \log \frac{a}{b}, \quad a_{i} \geq 0, b_{i} \geq 0, \quad a=\sum_{i=1}^{N} a_{i}, \quad b=\sum_{i=1}^{N} b_{i},
$$

where $a \log \frac{a}{0}=\infty$ if $a>0$, and $0 \log 0=0$. The equality holds if and only if $a_{i} / b_{i}=$ const.

\section{Iterative proportional scaling via decomposable sub- models}

In this section we propose a generalization of conventional IPS and study its properties. At each step of our procedure we update a larger set of marginals, which form a decomposable submodel. We prove convergence of our proposed procedure, if the amount of scaling is adjusted properly at each step. We also give a detailed analysis of our procedure when the current estimate is close to MLE.

\subsection{Proposed algorithms}

We now describe our proposed procedure. A model $M\left(\mathcal{C}^{\prime}\right)$ is a submodel of $M(\mathcal{C})$ if each generator of $M\left(\mathcal{C}^{\prime}\right)$ is contained in some generator of $M(\mathcal{C})$. Let $\left\{M\left(\mathcal{C}_{1}\right), \ldots, M\left(\mathcal{C}_{u}\right)\right\}$ be a set of decomposable submodels of $M(\mathcal{C})$ such that each generator of $M(\mathcal{C})$ is contained in the generating class of $M\left(\mathcal{C}_{j}\right)$ for some $j$. In this case we say that $\left\{M\left(\mathcal{C}_{1}\right), \ldots, M\left(\mathcal{C}_{u}\right)\right\}$ spans $M(\mathcal{C})$.

In our procedure there is a problem of normalization as discussed below. Therefore we denote the non-normalized estimated cell probability at the $t$-th step by $q^{(t)}$ and the normalized estimated cell probability by $p^{(t)}$.

Algorithm 1 Let $q^{(0)} \equiv 1 /|\mathcal{I}|$. We cycle through $\mathcal{C}_{1}, \mathcal{C}_{2}, \ldots, \mathcal{C}_{u}$ and for the $t$-th step we update the non-normalized estimated cell probabilities as follows

$$
q^{(t+1)}=q^{(t)} \frac{r_{j}}{q_{j}^{(t)}}, \quad j=(t \quad \bmod u)+1
$$

where $r_{j}$ is the maximum-entropy extension of the set of probability distributions $\{r[C]$ : $\left.C \in \mathcal{C}_{j}\right\}$ and $q_{j}^{(t)}$ is the maximum-entropy extension of the set of probability distributions $\left\{q^{(t)}[C]: C \in \mathcal{C}_{j}\right\}$, and the normalized cell probabilities as

$$
p^{(t+1)}(i)=\frac{q^{(t+1)}(i)}{\sum_{k \in \mathcal{I}} q^{(t+1)}(k)} .
$$


Example 3.1. Consider a 4-way contingency table $H \times J \times K \times L$ and the following hierarchical model with generating class $\mathcal{C}=\{\{H, J\},\{J, K\},\{K, L\},\{H, L\}\}$ ("4-cycle model") :

$$
p_{h j k l}=\exp \left(a_{h j}+b_{j k}+c_{k l}+d_{h l}\right) .
$$

By slight abuse of notation write $\Delta=\{H, J, K, L\}$. The following $\mathcal{C}_{1}$ and $\mathcal{C}_{2}$ is an example of the family of submodels that spans $\mathcal{C}$.

$$
\begin{aligned}
& \mathcal{C}_{1}=\{\{H, J\},\{J, K\},\{K, L\}\}, \\
& \mathcal{C}_{2}=\{\{H, J\},\{K, L\},\{H, L\}\} .
\end{aligned}
$$

For each submodel, the updating procedure is performed as follows.

$$
\begin{gathered}
q^{(t+1)}(i)=q^{(t)}(i) \times \frac{r\left(i_{h j}\right) \times r\left(i_{j k}\right) \times r\left(i_{k l}\right)}{r\left(i_{j}\right) \times r\left(i_{k}\right)} \times \frac{q^{(t)}\left(i_{j}\right) \times q^{(t)}\left(i_{k}\right)}{q^{(t)}\left(i_{h j}\right) \times q^{(t)}\left(i_{j k}\right) \times q^{(t)}\left(i_{k l}\right)}, \\
q^{(t+2)}(i)=q^{(t+1)}(i) \times \frac{r\left(i_{h j}\right) \times r\left(i_{k l}\right) \times r\left(i_{h l}\right)}{r\left(i_{h}\right) \times r\left(i_{l}\right)} \times \frac{q^{(t+1)}\left(i_{h}\right) \times q^{(t+1)}\left(i_{l}\right)}{q^{(t+1)}\left(i_{h j}\right) \times q^{(t+1)}\left(i_{k l}\right) \times q^{(t+1)}\left(i_{h l}\right)} .
\end{gathered}
$$

If we set $\mathcal{C}_{1}=\left\{C_{1}\right\}, \ldots, \mathcal{C}_{m}=\left\{C_{m}\right\}$, Algorithm 1 coincides with the conventional IPS. $\mathcal{C}_{1}, \ldots, \mathcal{C}_{m}$ span $\mathcal{C}$. Each $\mathcal{C}_{j}$ is composed of one generator of the model. Hence $M\left(\mathcal{C}_{j}\right)$ is a decomposable submodel of $M(\mathcal{C})$. Therefore Algorithm 1 is a generalization of conventional IPS. In the conventional IPS, $p^{(t+1)}(i)$ in (3) satisfies $p^{(t+1)}\left(i_{C}\right)=r\left(i_{C}\right)$, which is a likelihood equation in (11). But in general $p^{(t+1)}(i)$ in (5) does not satisfy (1). In other words, from a geometric viewpoint of $I$-projection in Csiszár ([4],[5]), the updating rule (41) is not a projection. We discuss it again in the next section.

In (4), we update $q^{(t)}(i)$. It should be noted that we have

$$
q^{(t)}(i) \times \frac{\prod_{C \in \mathcal{C}_{j}} r\left(i_{C}\right)}{\prod_{S \in \mathcal{S}_{j}} r\left(i_{S}\right)} \times \frac{\prod_{S \in \mathcal{S}_{j}} q^{(t)}\left(i_{S}\right)}{\prod_{C \in \mathcal{C}_{j}} q^{(t)}\left(i_{C}\right)}=p^{(t)}(i) \times \frac{\prod_{C \in \mathcal{C}_{j}} r\left(i_{C}\right)}{\prod_{S \in \mathcal{S}_{j}} r\left(i_{S}\right)} \times \frac{\prod_{S \in \mathcal{S}_{j}} p^{(t)}\left(i_{S}\right)}{\prod_{C \in \mathcal{C}_{j}} p^{(t)}\left(i_{C}\right)}
$$

because the normalizing constant is canceled on the right-hand side of (4). Also it is easy to see that, if Algorithm 1 in terms of $\left\{q^{(t)}(i)\right\}$ converges, then the limiting $q$ 's are automatically normalized.

Unfortunately it is difficult to prove convergence of Algorithm 1. The difficulty lies in the fact that the sum $\sum_{i \in \mathcal{I}} q^{(t+1)}(i)$ after updating might exceed 1 (i.e. $\left.\sum_{i \in \mathcal{I}} q^{(t+1)}(i)>1\right)$ in Algorithm 1 even if $q^{(t)}$ is normalized as $\sum_{i} q^{(t)}(i)=1$. However we recommend it because in practice, it works well and has converged to MLE in all of our experiments and converges faster than the conventional IPS as shown in Section 4.

In order to deal with the theoretical difficulty concerning the normalization of $q^{(t+1)}$ we consider adjusting the amount of updating. At this point, we need the following lemma.

Lemma 3.1. Let $r$ and $q$ be two probability distributions over $\mathcal{I}$, and $M\left(\mathcal{C}^{\prime}\right)$ a decomposable model over a nonempty (proper or improper) subset $\Delta^{\prime}$ of $\Delta$. Let $r_{\mathcal{C}^{\prime}}$ be the maximum-entropy extension of the set of probability distributions $\left\{r[C]: C \in \mathcal{C}^{\prime}\right\}$ and $q_{\mathcal{C}^{\prime}}$ 
be the maximum-entropy extension of the set of probability distributions $\left\{q[C]: C \in \mathcal{C}^{\prime}\right\}$. If $q\left[\Delta^{\prime}\right]$ is not an extension of the set of probability distributions $\left\{r[C]: C \in \mathcal{C}^{\prime}\right\}$, then there exists a unique $\alpha_{0} \geq 0$ for which the function

$$
q^{\prime}=q \times\left(\frac{r_{\mathcal{C}^{\prime}}}{q_{\mathcal{C}^{\prime}}}\right)^{\alpha}
$$

is a probability distribution.

Proof. In view of (2) we have

$$
1=\sum_{i \in \mathcal{I}} r_{\mathcal{C}^{\prime}}(i)=\sum_{i \in \mathcal{I}} q_{\mathcal{C}^{\prime}}(i)
$$

Therefore if

$$
\frac{r_{\mathcal{C}^{\prime}}(i)}{q_{\mathcal{C}^{\prime}}(i)} \leq 1
$$

for all $i$, then the equality in (6) holds for all $i$ with $q(i)>0$. Therefore under the condition of the lemma there exists at least one cell $i \in \mathcal{I}$ such that

$$
\frac{r_{\mathcal{C}^{\prime}}(i)}{q_{\mathcal{C}^{\prime}}(i)}>1, \quad q(i)>0 .
$$

Then $q(i)\left(r_{\mathcal{C}^{\prime}(i)} / q_{\mathcal{C}^{\prime}}(i)\right)^{\alpha}$ for this $i$ is strictly convex in $\alpha$ and diverges to $+\infty$ as $\alpha \rightarrow \infty$. Write

$$
g(\alpha)=\sum_{i \in \mathcal{I}} q(i)\left(\frac{r_{\mathcal{C}^{\prime}}(i)}{q_{\mathcal{C}^{\prime}}(i)}\right)^{\alpha} .
$$

Then $g(\alpha)$ is also strictly convex in $\alpha$ and diverges to $+\infty$ as $\alpha \rightarrow \infty$.

Write $\mathcal{C}^{\prime}=\left\{C_{1}, \ldots, C_{v}\right\}$ and $\mathcal{S}^{\prime}=\left\{S_{2}, \ldots, S_{v}\right\}$. Consider the differential of $g(\alpha)$ at $\alpha=0$.

$$
\begin{aligned}
g^{\prime}(0) & =\sum_{i} q(i) \log \left(\frac{\prod_{C \in \mathcal{C}^{\prime}} r\left(i_{C}\right)}{\prod_{S \in \mathcal{S}^{\prime}} r\left(i_{S}\right)} \times \frac{\prod_{S \in \mathcal{S}^{\prime}} q\left(i_{S}\right)}{\prod_{C \in \mathcal{C}^{\prime}} q\left(i_{C}\right)}\right) \\
& =\sum_{C \in \mathcal{C}^{\prime}} \sum_{i} q(i) \log \frac{r\left(i_{C}\right)}{q\left(i_{C}\right)}-\sum_{S \in \mathcal{S}^{\prime}} \sum_{i} q(i) \log \frac{r\left(i_{S}\right)}{q\left(i_{S}\right)} \\
& =\sum_{C \in \mathcal{C}^{\prime}} \sum_{i_{C}} q\left(i_{C}\right) \log \frac{r\left(i_{C}\right)}{q\left(i_{C}\right)}-\sum_{S \in \mathcal{S}^{\prime}} \sum_{i_{S}} q\left(i_{S}\right) \log \frac{r\left(i_{S}\right)}{q\left(i_{S}\right)} .
\end{aligned}
$$

Now

$$
\sum_{i_{C_{1}}} q\left(i_{C_{1}}\right) \log \frac{r\left(i_{C_{1}}\right)}{q\left(i_{C_{1}}\right)}
$$

is the negative of KL-divergence and nonpositive. By the log sum inequality,

$$
\sum_{i_{C_{k}}} q\left(i_{C_{k}}\right) \log \frac{r\left(i_{C_{k}}\right)}{q\left(i_{C_{k}}\right)}-\sum_{i_{S_{k}}} q^{(t)}\left(i_{S_{k}}\right) \log \frac{r\left(i_{S_{k}}\right)}{q\left(i_{S_{k}}\right)}
$$


is also nonpositive for $2 \leq k \leq v$. Equality holds if and only if

$$
r\left(i_{C}\right)=q^{(t)}\left(i_{C}\right), \quad \forall C \in \mathcal{C}^{\prime}, \forall i_{C} .
$$

Then, except for such a case, $g(0)=1, g^{\prime}(0)<0, g(\infty)=\infty$, and $g(\alpha)$ is strictly convex in $\alpha$. Therefore there exists a unique $\alpha_{0}>0$ such that $g\left(\alpha_{0}\right)=1$.

We now present the following algorithm and its modification based on Lemma 3.1.

Algorithm 2 Let $\alpha^{(t)} \geq 0$. We cycle through $\mathcal{C}_{1}, \mathcal{C}_{2}, \ldots, \mathcal{C}_{u}$ and for the $t$-th step we update the unnormalized estimated cell probabilities as

$$
q^{(t+1)}=q^{(t)} \times\left(\frac{r_{j}}{q_{j}^{(t)}}\right)^{\alpha^{(t)}}, \quad j=(t \quad \bmod u)+1,
$$

and the normalized cell probabilities as $p^{(t+1)}(i)=q^{(t+1)}(i) / \sum_{k \in \mathcal{I}} q^{(t+1)}(k)$.

Note that also in Algorithm 2 we do not need to normalize at each step and we can perform normalization any time, because $\left\{q^{(t)}(i)\right\}$ is always proportional to $\left\{p^{(t)}(i)\right\}$.

Algorithm 3 We cycle through $\mathcal{C}_{1}, \mathcal{C}_{2}, \ldots, \mathcal{C}_{u}$ and for the $t$-th step we update the estimated cell probabilities as follows

$$
q^{(t+1)}=q^{(t)} \times\left(\frac{r_{j}}{q_{j}^{(t)}}\right)^{\alpha_{0}^{(t)}}, \quad j=(t \quad \bmod u)+1,
$$

where $\alpha_{0}^{(t)} \geq 0$ is given in Lemma 3.1 with $q=q^{(t)}$.

\subsection{Correctness of the proposed algorithms}

In this section, we prove the correctness of proposed algorithms. As before let $\{r(i)\}$ denote the empirical distribution and let $\left\{p_{\mathcal{C}}(i)\right\}$ denote the MLE. Because we consider hierarchical models, the following equation holds ([4], [5]).

$$
I(r: q)=I\left(r: p_{\mathcal{C}}\right)+I\left(p_{\mathcal{C}}: q\right) .
$$

$I(r: q)$ corresponds to the log likelihood. Therefore we can prove the correctness of our algorithms by proving $I\left(p_{\mathcal{C}}: q^{(t)}\right) \rightarrow 0$ as $t \rightarrow \infty$.

Theorem 3.1. Algorithm 3 converges to MLE. 
Proof. Consider KL-divergence after updating,

$$
\begin{aligned}
I\left(p_{\mathcal{C}} ; p^{(t+1)}\right) & =\sum_{i} p_{\mathcal{C}}(i) \log \frac{p_{\mathcal{C}}(i)}{p^{(t+1)}(i)} \\
& =\sum_{i} p_{\mathcal{C}}(i) \log \frac{p_{\mathcal{C}}(i)}{p^{(t)}(i) \times\left(\frac{\prod_{C \in \mathcal{C}_{j}} r\left(i_{C}\right)}{\prod_{S \in \mathcal{S}_{j}} r\left(i_{S}\right)} \times \frac{\prod_{S \in \mathcal{S}_{j}} p^{(t)}\left(i_{S}\right)}{\prod_{C \in \mathcal{C}_{j}} p^{(t)}\left(i_{C}\right)}\right)^{\alpha_{0}^{(t)}}} \\
& =\sum_{i} p_{\mathcal{C}}(i) \log \frac{p_{\mathcal{C}}(i)}{p^{(t)}(i)}-\alpha_{0}^{(t)} \sum_{i} p_{\mathcal{C}}(i) \log \frac{\prod_{C \in \mathcal{C}_{j}} r\left(i_{C}\right)}{\prod_{S \in \mathcal{S}_{j}} r\left(i_{S}\right)} \times \frac{\prod_{S \in \mathcal{S}_{j}} p^{(t)}\left(i_{S}\right)}{\prod_{C \in \mathcal{C}_{j}} p^{(t)}\left(i_{C}\right)} .
\end{aligned}
$$

Write $\mathcal{C}_{j}=\left\{C_{1}, \ldots, C_{v}\right\}$ and $\mathcal{S}_{j}=\left\{S_{2}, \ldots, S_{v}\right\}$ as in the proof of Lemma 3.1. Then,

$$
\sum_{i} p_{\mathcal{C}}(i) \log \frac{r\left(i_{C_{1}}\right)}{p^{(t)}\left(i_{C_{1}}\right)}=\sum_{i_{C_{1}}} r\left(i_{C_{1}}\right) \log \frac{r\left(i_{C_{1}}\right)}{p^{(t)}\left(i_{C_{1}}\right)}
$$

is a KL-divergence, and nonnegative. By the log sum inequality,

$$
\sum_{i_{C_{k}}} r\left(i_{C_{k}}\right) \log \frac{r\left(i_{C_{k}}\right)}{p^{(t)}\left(i_{C_{k}}\right)}-\sum_{i_{S_{k}}} r\left(i_{S_{k}}\right) \log \frac{r\left(i_{S_{k}}\right)}{p^{(t)}\left(i_{S_{k}}\right)}
$$

is also nonnegative for $2 \leq k \leq v$. Therefore,

$$
I\left(p_{\mathcal{C}} ; p^{(t+1)}\right) \leq I\left(p_{\mathcal{C}} ; p^{(t)}\right)
$$

holds. Equality holds if and only if $r\left(i_{C}\right)=q^{(t)}\left(i_{C}\right), \forall C \in \mathcal{C}_{j}$. We see that $I\left(p_{\mathcal{C}} ; p^{(t)}\right)$ always decreases after updating. The rest of the proof is the same as the classical one $([14])$.

Corollary 3.1. Using $0<\alpha^{(t)} \leq \alpha_{0}^{(t)}$, Algorithm 2 converges to MLE.

Proof. Consider KL-divergence after updating,

$$
\begin{aligned}
& I\left(p_{\mathcal{C}} ; p^{(t+1)}\right) \\
& \quad=\sum_{i} p_{\mathcal{C}}(i) \log \frac{p_{\mathcal{C}}(i)}{q^{(t)}(i)}-\alpha^{(t)} \sum_{i} p_{\mathcal{C}}(i) \log \frac{\prod_{C \in \mathcal{C}_{j}} r\left(i_{C}\right)}{\prod_{S \in \mathcal{S}_{j}} r\left(i_{S}\right)} \times \frac{\prod_{S \in \mathcal{S}_{j}} q^{(t)}\left(i_{S}\right)}{\prod_{C \in \mathcal{C}_{j}} q^{(t)}\left(i_{C}\right)}+\log g\left(\alpha^{(t)}\right),
\end{aligned}
$$

where $g(\alpha)$ is given in (7) with $q=q^{(t)}$. Because $\alpha^{(t)} \leq \alpha_{0}^{(t)}, \log g\left(\alpha^{(t)}\right)$ is nonpositive and $I\left(p_{\mathcal{C}} ; p^{(t)}\right)$ always decreases after updating. The rest of the proof is the same as Theorem 3.1.

At this point we discuss Algorithm 3 from a geometric viewpoint of $I$-projection in the sense of Csiszár ([4], [5]). In our procedure we adjust a larger set of marginals than the conventional IPS and in practice KL-divergence decreases more in our proposed 
algorithms than the conventional IPS for each step. However it is difficult to guarantee this theoretically. The difficulty lies in the fact that the updating rule (9) is not a projection. In fact, if we repeat (9) twice with the same $\mathcal{C}_{j}$ then the cell probabilities change, whereas in the conventional IPS repeating the same updating step twice does not change the cell probabilities after the first update. We can understand the situation as follows. Starting from the current estimate $\left\{p^{(t)}(i)\right\}$ suppose that we repeat the step (9) with the same $\mathcal{C}_{j}$ until the cell probabilities converge to $\left\{p^{\star}(i)\right\}$. Then the limit $\left\{p^{\star}(i)\right\}$ maximizes the likelihood function among $\{p(i)\}$ of the form

$$
p(i)=p^{(t)}(i) \prod_{C \in \mathcal{C}_{j}} \mu\left(i_{C}\right) .
$$

The right-hand side of (10) forms a log-affine model through $\left\{p^{(t)}(i)\right\}$ (Section 4.2 .3 of [15]). Since updating a single $C \in \mathcal{C}_{j}$ in the conventional IPS is a special case of (10), it follows that

$$
I\left(p_{\mathcal{C}}: p^{\star}\right) \leq I\left(p_{\mathcal{C}}: p^{(t+1)^{\prime}}\right),
$$

where $\left\{p^{(t+1)^{\prime}}(i)\right\}$ is the updated estimate by the conventional IPS for some $C \in \mathcal{C}_{j}$. Therefore a larger decrease of KL-divergence of our procedure compared to conventional IPS is only guaranteed in the sense of (11). The situation will become more clear when we analyze the behavior of Algorithm 3 close to MLE in the next section.

\subsection{Analysis of behavior close to the maximum likelihood esti- mate}

In this section, we study the behavior of our algorithms when the current estimate is already close to MLE. We assume that MLE is in the interior of the parameter space and $p_{\mathcal{C}}(i)>0$ for all $i \in \mathcal{I}$. We analyze the behavior of $\alpha_{0}^{(t)}$. We also consider the value of $\alpha^{(t)}=\alpha_{1}^{(t)}$ which reduces the KL-divergence most and the value of $\alpha^{(t)}=\alpha_{2}^{(t)}$ such that KL-divergence decreases in Algorithm 2 for $0 \leq \alpha^{(t)} \leq \alpha_{2}^{(t)}$.

We repeatedly use the following expansion,

$$
\log (1+x)=x-\frac{x^{2}}{2}+O\left(x^{3}\right), \quad x \rightarrow 0
$$

Assume that the current estimate $\left\{p^{(t)}(i)\right\}$ is close to MLE in the following sense. For sufficiently small $\varepsilon>0$ and for all $C \in \mathcal{C}, S \in \mathcal{S}, i_{C}, i_{S}$ we have

$$
1-\varepsilon<\frac{r\left(i_{C}\right)}{p^{(t)}\left(i_{C}\right)}, \frac{r\left(i_{S}\right)}{p^{(t)}\left(i_{S}\right)}<1+\varepsilon .
$$

The following proposition describes the behavior of $\alpha_{0}^{(t)}$ in Algorithm 3 . 
Proposition 3.1. Assume $\left\{p^{(t)}(i)\right\}$ is close to MLE in the sense of (13)). Then

$$
\alpha_{0}^{(t)}=\frac{\sum_{i} p^{(t)}(i)\left\{\sum_{C \in \mathcal{C}_{j}}\left(\frac{r\left(i_{C}\right)}{p^{(t)}\left(i_{C}\right)}-1\right)^{2}-\sum_{S \in \mathcal{S}_{j}}\left(\frac{r\left(i_{S}\right)}{p^{(t)}\left(i_{S}\right)}-1\right)^{2}\right\}}{\sum_{i} p^{(t)}(i)\left\{\sum_{C \in \mathcal{C}_{j}}\left(\frac{r\left(i_{C}\right)}{p^{(t)}\left(i_{C}\right)}-1\right)-\sum_{S \in \mathcal{S}_{j}}\left(\frac{r\left(i_{S}\right)}{p^{(t)}\left(i_{S}\right)}-1\right)\right\}^{2}}+O(\varepsilon) .
$$

Before giving a proof of this Proposition we rewrite the numerator of the right-hand side of (14). Let $\mathcal{C}_{j}=\left\{C_{1}, \ldots, C_{v}\right\}$ and $\mathcal{S}_{j}=\left\{S_{2}, \ldots, S_{v}\right\}$. Then

$$
\begin{gathered}
\sum_{i} p^{(t)}(i)\left\{\sum_{C \in \mathcal{C}_{j}}\left(\frac{r\left(i_{C}\right)}{p^{(t)}\left(i_{C}\right)}-1\right)^{2}-\sum_{S \in \mathcal{S}_{j}}\left(\frac{r\left(i_{S}\right)}{p^{(t)}\left(i_{S}\right)}-1\right)^{2}\right\} \\
=\sum_{i_{C_{1}}} p^{(t)}\left(i_{C_{1}}\right)\left(\frac{r\left(i_{C_{1}}\right)}{p^{(t)}\left(i_{C_{1}}\right)}-1\right)^{2} \\
+\sum_{k=2}^{v} \sum_{i_{C_{k}}} p^{(t)}\left(i_{C_{k}}\right)\left(\frac{r\left(i_{C_{k}}\right)}{p^{(t)}\left(i_{C_{k}}\right)}-\frac{r\left(i_{S_{k}}\right)}{p^{(t)}\left(i_{S_{k}}\right)}\right)^{2} .
\end{gathered}
$$

Therefore the numerator is nonnegative. Also note that the denominator of the right-hand side of (14) can be written as

$$
\begin{aligned}
\sum_{C \in \mathcal{C}_{j}}\left(\frac{r\left(i_{C}\right)}{p^{(t)}\left(i_{C}\right)}-1\right)-\sum_{S \in \mathcal{S}_{j}}\left(\frac{r\left(i_{S}\right)}{p^{(t)}\left(i_{S}\right)}-1\right) \\
\quad=\left(\frac{r\left(i_{C_{1}}\right)}{p^{(t)}\left(i_{C_{1}}\right)}-1\right)+\sum_{k=2}^{v}\left(\frac{r\left(i_{C_{k}}\right)}{p^{(t)}\left(i_{C_{k}}\right)}-\frac{r\left(i_{S_{k}}\right)}{p^{(t)}\left(i_{S_{k}}\right)}\right) .
\end{aligned}
$$

We see that the numerator of $\alpha_{0}^{(t)}$ consists of the diagonal square terms when we expand the square of denominator in the form of (16). We now give a proof of Proposition 3.1.

Proof. Consider the following expansion,

$$
\begin{aligned}
\log \frac{\prod_{C \in \mathcal{C}_{j}} r\left(i_{C}\right)}{\prod_{S \in \mathcal{S}_{j}} r\left(i_{S}\right)} \times \frac{\prod_{S \in \mathcal{S}_{j}} p^{(t)}\left(i_{S}\right)}{\prod_{C \in \mathcal{C}_{j}} p^{(t)}\left(i_{C}\right)} & =\sum_{C \in \mathcal{C}_{j}} \log \frac{r\left(i_{C}\right)}{p^{(t)}\left(i_{C}\right)}-\sum_{S \in \mathcal{S}_{j}} \log \frac{r\left(i_{S}\right)}{p^{(t)}\left(i_{S}\right)} \\
& =\sum_{C \in \mathcal{C}_{j}}\left(\frac{r\left(i_{C_{j}}\right)}{p^{(t)}\left(i_{C}\right)}-1\right)-\sum_{S \in \mathcal{S}_{j}}\left(\frac{r\left(i_{S}\right)}{p^{(t)}\left(i_{S}\right)}-1\right)+O\left(\varepsilon^{2}\right) \\
& =O(\varepsilon) .
\end{aligned}
$$

Then the $s$-th derivative of $g\left(\alpha^{(t)}\right)$ at 0 is

$$
\begin{aligned}
g^{(s)}(0) & =\sum_{i} p^{(t)}(i)\left(\log \frac{\prod_{C \in \mathcal{C}_{j}} r\left(i_{C}\right)}{\prod_{S \in \mathcal{S}_{j}} r\left(i_{S}\right)} \times \frac{\prod_{S \in \mathcal{S}_{j}} p^{(t)}\left(i_{S}\right)}{\prod_{C \in \mathcal{C}_{j}} p^{(t)}\left(i_{C}\right)}\right)^{s} \\
& =O\left(\varepsilon^{s}\right) .
\end{aligned}
$$


The first and the second order derivatives of $g\left(\alpha^{(t)}\right)$ at 0 are,

$$
\begin{aligned}
g^{(1)}(0)= & \sum_{i} p^{(t)}(i)\left(\log \frac{\prod_{C \in \mathcal{C}_{j}} r\left(i_{C}\right)}{\prod_{S \in \mathcal{S}_{j}} r\left(i_{S}\right)} \times \frac{\prod_{S \in \mathcal{S}_{j}} p^{(t)}\left(i_{S}\right)}{\prod_{C \in \mathcal{C}_{j}} p^{(t)}\left(i_{C}\right)}\right) \\
= & \sum_{C \in \mathcal{C}_{j}} \sum_{i_{C}} p^{(t)}\left(i_{C}\right) \log \frac{r\left(i_{C}\right)}{p^{(t)}\left(i_{C}\right)}-\sum_{S \in \mathcal{S}_{j}} \sum_{i_{S}} p^{(t)}\left(i_{S}\right) \log \frac{r\left(i_{S}\right)}{p^{(t)}\left(i_{S}\right)} \\
= & \sum_{C \in \mathcal{C}_{j}} \sum_{i_{C}} p^{(t)}\left(i_{C}\right)\left\{\frac{r\left(i_{C}\right)}{p^{(t)}\left(i_{C}\right)}-1-\frac{1}{2}\left(\frac{r\left(i_{C}\right)}{p^{(t)}\left(i_{C}\right)}-1\right)^{2}\right\} \\
& -\sum_{S \in \mathcal{S}_{j}} \sum_{i_{S}} p^{(t)}\left(i_{S}\right)\left\{\frac{r\left(i_{S}\right)}{p^{(t)}\left(i_{S}\right)}-1-\frac{1}{2}\left(\frac{r\left(i_{S}\right)}{p^{(t)}\left(i_{S}\right)}-1\right)^{2}\right\}+O\left(\varepsilon^{3}\right) \\
= & \sum_{C \in \mathcal{C}_{j}} \sum_{i_{C}}\left\{\left(r\left(i_{C}\right)-p^{(t)}\left(i_{C}\right)\right)-\frac{p^{(t)}\left(i_{C}\right)}{2}\left(\frac{r\left(i_{C}\right)}{p^{(t)}\left(i_{C}\right)}-1\right)^{2}\right\} \\
& -\sum_{S \in \mathcal{S}_{j}} \sum_{i_{S}}\left\{\left(r\left(i_{S}\right)-p^{(t)}\left(i_{S}\right)\right)-\frac{p^{(t)}\left(i_{S}\right)}{2}\left(\frac{r\left(i_{S}\right)}{p^{(t)}\left(i_{S}\right)}-1\right)^{2}\right\}+O\left(\varepsilon^{3}\right) \\
= & \frac{1}{2} \sum_{i} p^{(t)}(i)\left\{\sum_{S \in \mathcal{S}_{j}}\left(\frac{r\left(i_{S}\right)}{p^{(t)}\left(i_{S}\right)}-1\right)^{2}-\sum_{C \in \mathcal{C}_{j}}\left(\frac{r\left(i_{C}\right)}{p^{(t)}\left(i_{C}\right)}-1\right)^{2}\right\}+O\left(\varepsilon^{3}\right),
\end{aligned}
$$

and

$$
\begin{aligned}
g^{(2)}(0) & =\sum_{i} p^{(t)}(i)\left(\log \frac{\prod_{C \in \mathcal{C}_{j}} r\left(i_{C}\right)}{\prod_{S \in \mathcal{S}_{j}} r\left(i_{S}\right)} \times \frac{\prod_{S \in \mathcal{S}_{j}} p^{(t)}\left(i_{S}\right)}{\prod_{C \in \mathcal{C}_{j}} p^{(t)}\left(i_{C}\right)}\right)^{2} \\
& =\sum_{i} p^{(t)}(i)\left\{\sum_{C \in \mathcal{C}_{j}}\left(\frac{r\left(i_{C}\right)}{p^{(t)}\left(i_{C}\right)}-1\right)-\sum_{S \in \mathcal{S}_{j}}\left(\frac{r\left(i_{S}\right)}{p^{(t)}\left(i_{S}\right)}-1\right)\right\}^{2}+O\left(\varepsilon^{3}\right) .
\end{aligned}
$$

Then, we expand $g\left(\alpha^{(t)}\right)$ at 0 ,

$$
g\left(\alpha^{(t)}\right)=g(0)+\alpha^{(t)} g^{(1)}(0)+\frac{\left(\alpha^{(t)}\right)^{2}}{2} g^{(2)}(0)+O\left(\varepsilon^{3}\right) .
$$

Assuming normalization at each step of the algorithm, we have $g(0)=1$ and substituting $\alpha_{0}^{(t)}$ for $\alpha^{(t)}$, we obtain

$$
\begin{aligned}
\alpha_{0}^{(t)} & =\frac{-2 g^{(1)}(0)}{g^{(2)}(0)}+O(\varepsilon) \\
& =\frac{\sum_{i} p^{(t)}(i)\left\{\sum_{C \in \mathcal{C}_{j}}\left(\frac{r\left(i_{C}\right)}{p^{(t)}\left(i_{C}\right)}-1\right)^{2}-\sum_{S \in \mathcal{S}_{j}}\left(\frac{r\left(i_{S}\right)}{p^{(t)}\left(i_{S}\right)}-1\right)^{2}\right\}}{\sum_{i} p^{(t)}(i)\left\{\sum_{C \in \mathcal{C}_{j}}\left(\frac{r\left(i_{C}\right)}{p^{(t)}\left(i_{C}\right)}-1\right)-\sum_{S \in \mathcal{S}_{j}}\left(\frac{r\left(i_{S}\right)}{p^{(t)}\left(i_{S}\right)}-1\right)\right\}^{2}}+O(\varepsilon) .
\end{aligned}
$$


Consider (15) and (16). If the signs of the terms on the right hand side of (16) are "random" then we can expect that $\alpha_{0}^{(t)}$ is close to 1 . We can imagine that $\left\{p^{(t)}(i)\right\}$ converges to MLE from various directions. Then $\alpha_{0}^{(t)}$ is close to 1 "on the average". Furthermore as shown in the following proposition $\alpha_{0}^{(t)}$ is the optimum value of the adjustment close to MLE. We believe that this is the reason that Algorithm 1 works very well in practice.

Proposition 3.2. Assume $\left\{p^{(t)}(i)\right\}$ is close to MLE in the sense of (13)). Then

$$
\alpha_{1}^{(t)}=\alpha_{0}^{(t)}+O(\varepsilon),
$$

where $\alpha_{1}^{(t)}$ is the value of $\alpha^{(t)}$ which reduces the KL-divergence most.

Proof. Define $F\left(\alpha^{(t)}\right)$ by

$$
F\left(\alpha^{(t)}\right)=\alpha^{(t)} \sum_{i} p^{*}(i) \log \frac{\prod_{C \in \mathcal{C}_{j}} r\left(i_{C}\right)}{\prod_{S \in \mathcal{S}_{j}} r\left(i_{S}\right)} \times \frac{\prod_{S \in \mathcal{S}_{j}} p^{(t)}\left(i_{S}\right)}{\prod_{C \in \mathcal{C}_{j}} p^{(t)}\left(i_{C}\right)},
$$

which corresponds to the decrease of KL-divergence before normalization. Consider the derivative of $F\left(\alpha^{(t)}\right)$,

$$
\begin{aligned}
F^{(1)}\left(\alpha^{(t)}\right)= & \sum_{i} p^{*}(i) \log \frac{\prod_{C \in \mathcal{C}_{j}} r\left(i_{C}\right)}{\prod_{S \in \mathcal{S}_{j}} r\left(i_{S}\right)} \times \frac{\prod_{S \in \mathcal{S}_{j}} p^{(t)}\left(i_{S}\right)}{\prod_{C \in \mathcal{C}_{j}} p^{(t)}\left(i_{C}\right)} \\
= & g^{(1)}(0)+\sum_{i} p^{(t)}(i)\left(\frac{p^{*}(i)}{p^{(t)}(i)}-1\right) \log \frac{\prod_{C \in \mathcal{C}_{j}} r\left(i_{C}\right)}{\prod_{S \in \mathcal{S}_{j}} r\left(i_{S}\right)} \times \frac{\prod_{S \in \mathcal{S}_{j}} p^{(t)}\left(i_{S}\right)}{\prod_{C \in \mathcal{C}_{j}} p^{(t)}\left(i_{C}\right)} \\
= & g^{(1)}(0)+\sum_{i} p^{(t)}(i)\left(\frac{p^{*}(i)}{p^{(t)}(i)}-1\right)\left\{\sum_{C \in \mathcal{C}_{j}}\left(\frac{r\left(i_{C}\right)}{p^{(t)}\left(i_{C}\right)}-1\right)-\sum_{S \in \mathcal{S}_{j}}\left(\frac{r\left(i_{S}\right)}{p^{(t)}\left(i_{S}\right)}-1\right)\right\} \\
& +O\left(\varepsilon^{3}\right) \\
= & g^{(1)}(0)+\sum_{i} p^{(t)}(i)\left\{\sum_{C \in \mathcal{C}_{j}}\left(\frac{r\left(i_{C}\right)}{p^{(t)}\left(i_{C}\right)}-1\right)^{2}-\sum_{S \in \mathcal{S}_{j}}\left(\frac{r\left(i_{S}\right)}{p^{(t)}\left(i_{S}\right)}-1\right)^{2}\right\}+O\left(\varepsilon^{3}\right) \\
= & -g^{(1)}(0)+O\left(\varepsilon^{3}\right) .
\end{aligned}
$$

Consider the derivative of $F\left(\alpha^{(t)}\right)-\log g\left(\alpha^{(t)}\right)$ and equating 0 , we obtain,

$$
F^{(1)}\left(\alpha_{1}^{(t)}\right)-\frac{g^{(1)}\left(\alpha_{1}^{(t)}\right)}{g\left(\alpha_{1}^{(t)}\right)}=0 .
$$

Then

$$
\begin{aligned}
g^{(1)}\left(\alpha_{1}^{(t)}\right) & =g^{(1)}(0)+\alpha_{1}^{(t)} g^{(2)}(0)+O\left(\varepsilon^{3}\right), \\
g\left(\alpha_{1}^{(t)}\right) & =g(0)+\alpha_{1}^{(t)} g^{(1)}(0)+\frac{\left(\alpha_{1}^{(t)}\right)^{2}}{2} g^{(2)}(0)+O\left(\varepsilon^{3}\right) \\
& =1+O\left(\varepsilon^{2}\right)
\end{aligned}
$$


and

$$
F^{(1)}\left(\alpha_{1}^{(t)}\right)-g^{(1)}\left(\alpha_{1}^{(t)}\right)+O\left(\varepsilon^{3}\right)=-g^{(1)}(0)-g^{(1)}(0)-\alpha_{1}^{(t)} g^{(2)}(0)+O\left(\varepsilon^{3}\right)=0 .
$$

Therefore we have

$$
\alpha_{1}^{(t)}=\frac{-2 g^{(1)}(0)}{g^{(2)}(0)}+O(\varepsilon)=\alpha_{0}^{(t)}+O(\varepsilon)
$$

Finally we show that KL-divergence decreases in the range $0<\alpha^{(t)}<2 \alpha_{0}^{(t)}$. This result indicates that in Algorithm 2, $\alpha^{(t)}>\alpha_{0}^{(t)}$ often decreases KL-divergence in practice.

Proposition 3.3. Assume $\left\{p^{(t)}(i)\right\}$ is close to MLE in the sense of (13). Then

$$
\alpha_{2}^{(t)}=2 \alpha_{0}^{(t)}+O(\varepsilon) .
$$

where $\alpha_{2}^{(t)}$ is the value of $\alpha^{(t)}$ such that $I\left(p_{\mathcal{C}}: p^{(t+1)}\right)=I\left(p_{\mathcal{C}}: p^{(t)}\right)$ in Algorithm 2 .

Proof.

$$
0=F\left(\alpha_{2}^{(t)}\right)-\log g\left(\alpha_{2}^{(t)}\right)=-\alpha_{2}^{(t)} g^{(1)}(0)-\alpha_{2}^{(t)} g^{(1)}(0)+\frac{\left(\alpha_{2}^{(t)}\right)^{2}}{2} g^{(2)}(0)+O\left(\varepsilon^{3}\right)
$$

and

$$
\alpha_{2}^{(t)}=\frac{-4 g^{(1)}(0)}{g^{(2)}(0)}+O(\varepsilon)=2 \alpha_{0}^{(t)}+O(\varepsilon)
$$

We show the behavior of $\log g\left(\alpha^{(t)}\right)$ and $F\left(\alpha^{(t)}\right)-\log g\left(\alpha^{(t)}\right)$ in Figure 1. Proposition 3.1. Proposition 3.2 and Proposition 3.3 indicate that in many cases we can decrease KL-divergence by using $\alpha^{(t)}=1$. In the next section we illustrate this by numerical experiments.

\section{Numerical experiments for cycle models}

In this section, we compare our Algorithm 1 with the conventional IPS by numerical experiments. We consider $J$-way cycle model with the generating class $\{\{1,2\},\{2,3\}, \ldots,\{J-$ $1, J\},\{J, 1\}\}$ for $J \geq 4$. As a family of decomposable submodels which span the model we use the set of two decomposable submodels obtained by deleting one element of generating class of the hierarchical model. We show the considered model and its submodels in Table 1, where $\{1,2\}$ is abbreviated as 12 . For example in the 5-way case we span $M_{5}=\{12,23,34,45,15\}$ by $M_{5} \backslash\{15\}$ and $M_{5} \backslash\{23\}$ as illustrated in Figure 2,

Before we present the results of the experiments, we consider the space-saving implementation of Algorithm 1. 


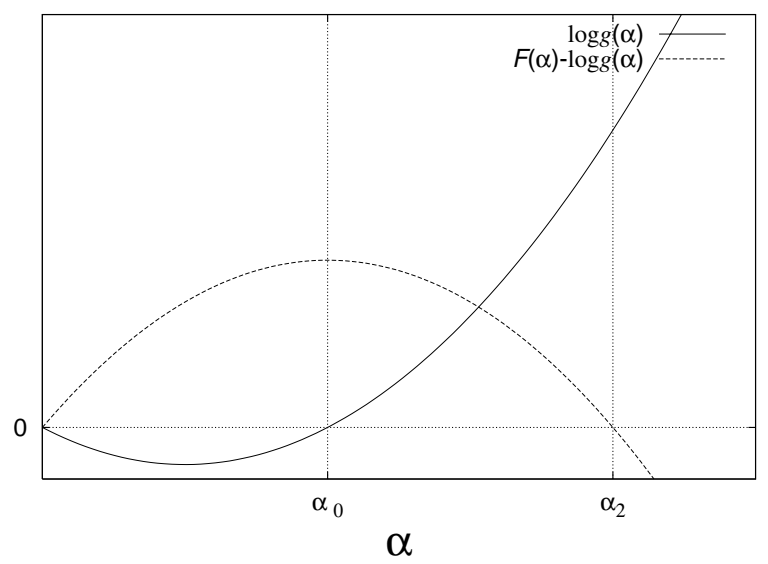

Figure 1: Behavior of $\log g\left(\alpha^{(t)}\right)$ and $F\left(\alpha^{(t)}\right)-\log g\left(\alpha^{(t)}\right)$

Table 1: The submodels in numerical experiments

\begin{tabular}{|c|c|c|}
\hline Dim & Hierarchical model & Decomposable submodels \\
\hline 4 & $M_{4}=\{12,23,34,14\}$ & $M_{4} \backslash\{14\}, M_{4} \backslash\{23\}$ \\
\hline 5 & $M_{5}=\{12,23,34,45,15\}$ & $M_{5} \backslash\{15\}, M_{5} \backslash\{23\}$ \\
\hline 6 & $M_{6}=\{12,23,34,45,56,16\}$ & $M_{6} \backslash\{16\}, M_{6} \backslash\{34\}$ \\
\hline 7 & $M_{7}=\{12,23,34,45,56,67,17\}$ & $M_{7} \backslash\{17\}, M_{7} \backslash\{34\}$ \\
\hline 8 & $M_{8}=\{12,23,34,45,56,67,78,18\}$ & $M_{8} \backslash\{18\}, M_{8} \backslash\{45\}$ \\
\hline
\end{tabular}
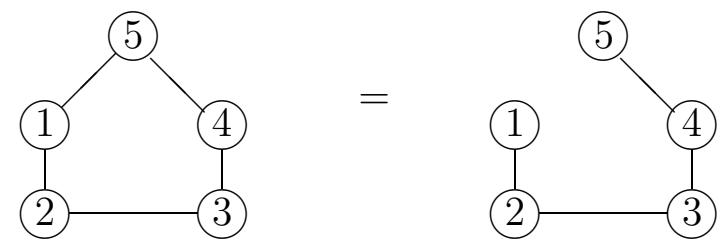

$\cup$

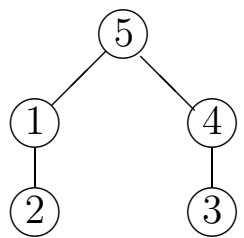

Figure 2: A decomposable submodels in a 5-way case

\subsection{Tree-computation of Algorithm 1}

For the conventional IPS, the implementation of the tree-computation has been considered in Jiroušek [12] and Jiroušek and Přeučil [13. Badsberg and Malvestuto[1] improved the algorithm by applying the Markovian information propagation techniques with junction trees of the triangulated models.

In this section we apply the Markovian propagation approach to our Algorithm 1 for cycle models. We triangulate the $J$-way cycle model by adding the edges $\{1,3\},\{1,4\}, \ldots,\{1, J-$ $1\}$. Let $\mathcal{D}^{*}$ and $\mathcal{S}^{*}$ denote the triangulated model and the set of separators of $\mathcal{D}^{*}$,

$$
\mathcal{D}^{*}=\{\{1,2,3\},\{1,3,4\}, \ldots,\{1, J-1, J\}\}, \quad \mathcal{S}^{*}=\{\{1,3\},\{1,4\}, \ldots,\{1, J-1\}\} .
$$


Then we note that the cell probabilities $p(i)$ satisfy

$$
p(i)=\frac{\prod_{C \in \mathcal{D}^{*}} p\left(i_{C}\right)}{\prod_{S \in \mathcal{S}^{*}} p\left(i_{S}\right)} .
$$

So we consider the tree-computation algorithm which updates $q^{(t)}\left(i_{C}\right), C \in \mathcal{D}^{*}$ instead of $q^{(t)}(i)$. Let $\left|\mathcal{I}_{C}^{*}\right|=\max _{C \in \mathcal{C}^{*}}\left|\mathcal{I}_{C}\right|$. While the computational cost per an update procedure of Algorithm 1 is $O(|\mathcal{I}|)$, that of the tree-computation algorithm is reduced to $O\left(\left|\mathcal{I}_{C}^{*}\right|\right)$. Denote by $C_{j}^{*}$ a generator $\{1, j-1, j\}, j=1, \ldots, J$. The junction tree for $\mathcal{D}^{*}$ is uniquely defined as in Figure 4.1. The decomposable submodels we use are $M_{J}^{1}=M_{J} \backslash\{J-1, J\}$ and $M_{J}^{2}=M_{J} \backslash\left\{J^{\prime}-1, J^{\prime}\right\}$ for some $1<J^{\prime}<J$. Direct the junction tree in two ways such that $C_{J}$ and $C_{J^{\prime}}$ are the unique sink as in Figure 3 and denote them by $T_{1}$ and $T_{2}$, respectively. Then the Markovian propagation algorithm proposed here is described as information propagation on $T_{1}$ and $T_{2}$.

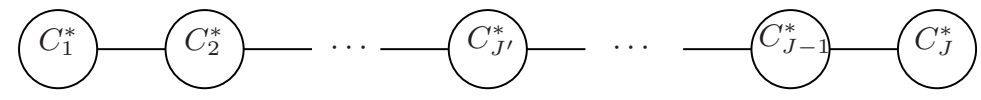

Figure 3: The junction tree for the $J$-way cycle model

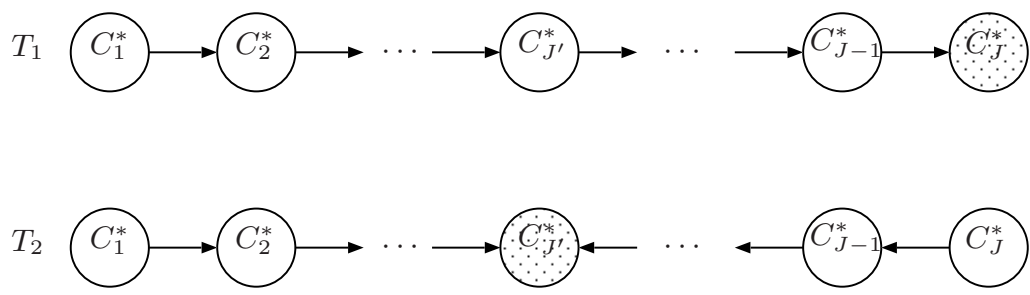

Figure 4: The directed trees

\section{Algorithm 4}

Define $r^{(t)}\left(i_{\{1,2\}}\right)=r\left(i_{\{1,2\}}\right)$ and $r^{(t)}\left(i_{\{J-1, J\}}\right)=r\left(i_{\{J-1, J\}}\right)$ for all $t$

(1) Update via $M_{J}^{1}$

for $j=3$ to $J$ do

if $j \neq J$, update $q^{(t)}\left(i_{C_{j}^{*}}\right)$ and $r^{(t)}\left(i_{\{1, j\}}\right)$ by

$$
q^{(t+1)}\left(i_{C_{j}^{*}}\right)=q^{(t)}\left(i_{C_{j}^{*}}\right) \times \frac{r^{(t+1)}\left(i_{\{1, j-1\}}\right) r\left(i_{\{j-1, j\}}\right)}{r\left(i_{\{j-1\}}\right)} \times \frac{q^{(t)}\left(i_{\{j-1\}}\right)}{q^{(t)}\left(i_{\{1, j-1\}}\right) \times q^{(t)}\left(i_{\{j-1, j\}}\right)},
$$




$$
r^{(t+1)}\left(i_{\{1, j\}}\right)=\sum_{i_{j-1}} q^{(t+1)}\left(i_{C_{j}^{*}}\right)
$$

and send $r^{(t)}\left(i_{\{1, j\}}\right)$ to $C_{j+1}^{*}$.

if $j=J$, update $q^{(t)}\left(i_{C_{J}^{*}}\right)$ by (20) .

(2) Update via $M_{J}^{2}$

for $j=3$ to $J^{\prime}-1$

update $q^{(t)}\left(i_{C_{j}^{*}}\right)$ and $r^{(t)}\left(i_{\{1, j\}}\right)$ by (20) and send $r^{(t)}\left(i_{\{1, j\}}\right)$ to $C_{j+1}^{*}$.

for $j=J$ to $J^{\prime}+1$

update $q^{(t)}\left(i_{C_{j}^{*}}\right)$ and $r^{(t)}\left(i_{\{1, j-1\}}\right)$ by

$$
\begin{gathered}
q^{(t+1)}\left(i_{C_{j}^{*}}\right)=q^{(t)}\left(i_{C_{j}^{*}}\right) \times \frac{r^{(t+1)}\left(i_{\{1, j\}}\right) r\left(i_{\{j-1, j\}}\right)}{r\left(i_{\{j\}}\right)} \times \frac{q^{(t)}\left(i_{\{j\}}\right)}{q^{(t)}\left(i_{\{1, j\}}\right) q^{(t)}\left(i_{\{j-1, j\}}\right)}, \\
r^{(t+1)}\left(i_{\{1, j-1\}}\right)=\sum_{i_{j}} q^{(t+1)}\left(i_{C_{j}^{*}}\right)
\end{gathered}
$$

and send $r^{(t)}\left(i_{\{1, j-1\}}\right)$ to $C_{j-1}^{*}$.

Update $q^{(t)}\left(i_{C_{J^{\prime}}^{*}}\right)$ by

$$
q^{(t+1)}\left(i_{C_{J^{\prime}}^{*}}\right)=q^{(t)}\left(i_{C_{J^{\prime}}^{*}}\right) \times \frac{r^{(t+1)}\left(i_{\left\{1, J^{\prime}-1\right\}}\right) r^{(t+1)}\left(i_{\left\{1, J^{\prime}\right\}}\right)}{r\left(i_{\{1\}}\right)} \times \frac{q^{(t)}\left(i_{\{1\}}\right)}{q^{(t)}\left(i_{\left\{1, J^{\prime}-1\right\}}\right) q^{(t)}\left(i_{\{1, J\}}\right)} .
$$

It is easy to show that

$$
\begin{aligned}
q^{(t+1)}(i) & =\frac{\prod_{C \in \mathcal{D}^{*}} q^{(t+1)}\left(i_{C}\right)}{\prod_{S \in \mathcal{S}^{*}} q^{(t+1)}\left(i_{S}\right)} \\
& =\frac{\prod_{C \in \mathcal{D}^{*}} q^{(t)}\left(i_{C}\right)}{\prod_{S \in \mathcal{S}^{*}} q^{(t)}\left(i_{S}\right)} \times \frac{\prod_{C \in \mathcal{C}_{1}} r\left(i_{C}\right)}{\prod_{S \in \mathcal{S}_{1}} r\left(i_{S}\right)} \times \frac{\prod_{S \in \mathcal{S}_{1}} q^{(t)}\left(i_{S}\right)}{\prod_{C \in \mathcal{C}_{1}} q^{(t)}\left(i_{C}\right)} \\
& =q^{(t)}(i) \times \frac{\prod_{C \in \mathcal{C}_{1}} r\left(i_{C}\right)}{\prod_{S \in \mathcal{S}_{1}} r\left(i_{S}\right)} \times \frac{\prod_{S \in \mathcal{S}_{1}} q^{(t)}\left(i_{S}\right)}{\prod_{C \in \mathcal{C}_{1}} q^{(t)}\left(i_{C}\right)} .
\end{aligned}
$$

(21) looks the same as (44). However there are some minor differences. In (44) $q^{(t)}\left(i_{S}\right)=$ $\sum_{i_{\Delta \backslash S}} q^{(t)}(i)$. However $q^{(t)}\left(i_{S}\right)$ in (21) is derived by $q^{(t)}\left(i_{S}\right)=\sum_{i_{C \backslash S}} q^{(t)}\left(i_{C}\right)$ for some $C \in \mathcal{C}^{*}$. Since $q^{(t)}\left(i_{S}\right)$ is not necessarily normalized, $\sum_{i_{C \backslash S}} q^{(t)}\left(i_{C}\right) \neq \sum_{i_{C^{\prime} \backslash S}} q^{(t)}\left(i_{C^{\prime}}\right)$ for $C \neq C^{\prime}$ and $C, C^{\prime} \in \mathcal{C}^{*}$. Hence in general $\sum_{i_{C \backslash S}} q^{(t)}\left(i_{C}\right) \neq \sum_{i_{\Delta \backslash S}} q^{(t)}(i)$. In this sense Algorithm 4 is an approximate algorithm for Algorithm 1.

In the experiments, we compare the performance of Algorithm 4 and the Markovian propagation algorithm for the conventional IPS by Badsberg and Malvestuto[1]. 


\subsection{The results of the numerical experiments}

In this section we present the results of numerical experiments. We set $I_{1}=\cdots=I_{\delta}=I$ and $I=2,3$ or 4 . We generated random contingency tables by filling each cell by uniform random integers from 1 to $10^{6}$ and we obtained MLE by Algorithm 4 and the Markovian propagation algorithm for the conventional IPS by Badsberg and Malvestuto[1]. As the convergence criterion we used

$$
\sum_{j=3}^{J} \sum_{i_{C_{j}^{*}}^{*} \in \mathcal{I}_{C_{j}^{*}}}\left|q^{(t+1)}\left(i_{C_{j}}^{*}\right)-q^{(t)}\left(i_{C_{j}}^{*}\right)\right| \leq 10^{-6} .
$$

For each dimension and each number of levels, we generated 1000 contingency tables and took the average of the $\mathrm{CPU}$ time and the number of steps to convergence. Denote by $\tau$ and $\tau_{\text {conv }}$ the CPU time for Algorithm 4 and the conventional IPS, respectively. Let $\nu$ and $\nu_{\text {conv }}$ be the number of steps to convergence for Algorithm 4 and the conventional IPS, respectively. We also calculated the probability that $\tau<\tau_{\text {conv }}$ and $\nu / \nu_{\text {conv }}$. The computation was done on a Pentium IV 3.2GHz CPU machine.

The results are shown in Table 2, In all of our runs Algorithm 4 converged to MLE. The experiments show that Algorithm 4 converges faster when the dimension is larger than 7. The computational cost per an update of Algorithm 4 is expected to be larger than that of the conventional IPS. As we can see from Table 2, however, the number of steps to convergence of Algorithm 4 is smaller than that of the conventional IPS. $\nu / \nu_{\text {conv }}$ gets smaller as the dimension of the model gets larger. Therefore the results of the experiments suggest that Algorithm 4 is more efficient than the conventional IPS when the dimension of the model is large for general hierarchical models.

\section{Some discussions}

For using the proposed algorithms, we have to find a family of decomposable submodels that span a generating class of a hierarchical model. We recommend spanning the generating class by a small number of large decomposable submodels. Here large decomposable submodels might mean maximal submodels in the sense of model inclusion or submodels with largest degrees of freedom. In the literature some methods for finding a maximal chordal subgraph of a given graph are studied ([2], 7], [19]). In the case of graphical models, this might give a solution to our problem. However we have to satisfy the condition that each element of a generating class is contained in at least one decomposable submodel. Therefore we need a method to find a maximal chordal subgraph under the restriction that specific generators are contained.

A referee suggested the following simple algorithm. Suppose that a model $M(\mathcal{C})$ with $|\mathcal{C}|=m$ is given. For each set $C$ in $\mathcal{C}$ choose an ordering $\left(C_{1}, \ldots C_{m}\right)$ of sets in $\mathcal{C}$ such that $C_{1}=C$ and $\left|C_{j-1} \cap C_{j}\right|=\max$ for all $j \geq 2$; set $C^{\prime}=\left\{C_{1}\right\}$; 
Table 2: CPU time and the number of steps to convergence

(i) $I=2$

\begin{tabular}{|c|cc|c|cc|c|}
\hline \multirow{2}{*}{$\operatorname{Dim}$} & \multicolumn{2}{|c|}{ CPU time } & $\operatorname{Pr}\left(\tau<\tau_{\text {conv }}\right)$ & \multicolumn{3}{|c|}{ number of steps } \\
\cline { 2 - 7 } & $\tau_{\text {conv }}$ & $\tau$ & & $\nu_{\text {conv }}$ & $\nu$ & $\nu / \nu_{\text {conv }}$ \\
\hline 4 & 0.0156 & 0.0178 & 0.171 & 11.652 & 6.887 & 0.591 \\
5 & 0.0193 & 0.0205 & 0.237 & 9.391 & 4.413 & 0.470 \\
6 & 0.0233 & 0.0198 & 0.465 & 7.841 & 3.348 & 0.427 \\
7 & 0.0289 & 0.0204 & 0.884 & 8.000 & 3.000 & 0.375 \\
8 & 0.0407 & 0.0258 & 0.957 & 9.000 & 3.000 & 0.333 \\
\hline
\end{tabular}

(ii) $I=3$

\begin{tabular}{|c|cc|c|cc|c|}
\hline \multirow{2}{*}{$\operatorname{Dim}$} & \multicolumn{2}{|c|}{ CPU time } & $\operatorname{Pr}\left(\tau<\tau_{\text {conv }}\right)$ & \multicolumn{3}{|c|}{ number of steps } \\
\cline { 2 - 7 } & $\tau_{\text {conv }}$ & $\tau$ & & $\nu_{\text {conv }}$ & $\nu$ & $\nu / \nu_{\text {conv }}$ \\
\hline 4 & 0.0337 & 0.0440 & 0.023 & 11.098 & 6.428 & 0.579 \\
5 & 0.0455 & 0.0463 & 0.394 & 9.345 & 4.455 & 0.477 \\
6 & 0.0484 & 0.0451 & 0.469 & 7.000 & 3.000 & 0.429 \\
7 & 0.0697 & 0.0559 & 0.929 & 8.000 & 3.000 & 0.375 \\
8 & 0.0951 & 0.0672 & 0.997 & 9.000 & 3.000 & 0.333 \\
\hline
\end{tabular}

(iii) $I=4$

\begin{tabular}{|c|cc|c|cc|c|}
\hline \multirow{2}{*}{$\operatorname{Dim}$} & \multicolumn{2}{|c|}{ CPU time } & $\operatorname{Pr}\left(\tau<\tau_{\text {conv }}\right)$ & \multicolumn{3}{|c|}{ number of steps } \\
\cline { 2 - 7 } & $\tau_{\text {conv }}$ & $\tau$ & & $\nu_{\text {conv }}$ & $\nu$ & $\nu / \nu_{\text {conv }}$ \\
\hline 4 & 0.0665 & 0.0941 & 0.000 & 10.493 & 4.943 & 0.471 \\
5 & 0.0722 & 0.1032 & 0.041 & 7.080 & 2.980 & 0.421 \\
6 & 0.1005 & 0.1007 & 0.324 & 7.000 & 3.000 & 0.429 \\
7 & 0.1437 & 0.1254 & 0.971 & 8.000 & 3.000 & 0.375 \\
8 & 0.2028 & 0.1551 & 0.997 & 9.000 & 3.000 & 0.333 \\
\hline
\end{tabular}

for $j=2, \ldots, m$

if $\mathcal{C}^{\prime} \cup\left\{C_{j}\right\}$ has the running intersection property then set $\mathcal{C}^{\prime}:=\mathcal{C}^{\prime} \cup\left\{C_{j}\right\}$.

Note that testing the running intersection property on a set family takes linear time [18].

In this paper we compared various algorithms of IPS in terms of the CPU time to convergence. We showed that proposed algorithm converges faster than conventional IPS when the model is large by numerical experiments. We consider the implementation of the tree-computation of Algorithm 1 only in the case of cycle models. It may be possible to implement the tree-computation of Algorithm 1 for general hierarchical model when the decomposable submodels are given. This topic needs further investigation and is left to our future research.

Acknowledgment. The authors are grateful to Hisayuki Hara for implementation of the tree-computation in Section 4 and to Satoshi Kuriki for very useful comments. They thank two referees for very constructive and detailed comments. 


\section{References}

[1] Badsberg, J. H. and Malvestuto, F. M. (2001), An implementation of the iterative proportional fitting procedure by propagation trees. Computational Statistics \& Data Analysis, Vol.37, pp.297-322.

[2] Berry, A.,Heggernes, P. and Villanger, Y. (2003), A vertex incremental approach for dynamically maintaining chordal graphs. Lecture Notes in Computer Science, Vol.2906, pp.47-57.

[3] Cover, T. M. and Thomas, J. A. (2006). Elements of Information Theory. 2nd ed. Wiley, New York.

[4] Csiszár, I. (1975), I-divergence geometry of probability distributions and minimization problems. Ann. Probab., Vol.3, pp.146-158.

[5] Csiszár, I. (1989), A geometric interpretation of Darroch and Ratcliff's generalized iterative scaling. Ann. Stat., Vol.17, pp.1409-1413.

[6] Darroch, J. N. and Ratcliff, D. (1972), Generalized iterative scaling for log-linear models. Ann. Math. Statist., Vol.43, pp.1470-1480.

[7] Dearing, P. M., Shier, D. R. and Warner, D. D. (1988), Maximal chordal subgraphs. Disc. Appl. Math., Vol.20, pp.181-190.

[8] Deming, W. E. and Stephan, F. F. (1940), On a least squares adjustment of a sampled frequency table when the expected marginal totals are known. Ann. Math. Statist., Vol.11, pp.427-444.

[9] Denteneer, D. and Verbeek, A. (1986), A fast algorithm for iterative proportional fitting in log-linear models. Computational Statistics and Data Analysis, Vol.3, pp.251264.

[10] Fienberg, S. E. (1970), An iterative procedure for estimation in contingency tables. Ann. Math. Statist., Vol.41, pp.907-917.

[11] Ireland, C. T. and Kullback, S. (1968), Contingency tables with given marginals. Biometrika, Vol.55, pp.179-188.

[12] Jiroušek, R. (1991), Solution of the marginal problem and decomposable distributions. Kybernetika, Vol.27, pp.403-412.

[13] Jiroušek, R. and Přeučil, S. (1995), On the effective implementation of the iterative proportional fitting procedure. Computational Statistics and Data Analysis, Vol.19, pp.177-189.

[14] Kullback, S. (1968), Probability densities with given marginals. Ann. Math. Statist., Vol.39, pp.1236-1243. 
[15] Lauritzen, S. L. (1997), Graphical Models. Clarendon Press, Oxford.

[16] Malvestuto, F. M. (1989), Computing the maximum-entropy extension of given discrete probability distributions. Computational Statistics and Data Analysis, Vol.8, pp.299-311.

[17] Rüschendorf, L. (1995), Convergence of the iterative proportional fitting procedure. Ann. Stat., Vol.23, pp.1160-1174.

[18] Tarjan, R. E. and Yannakakis, M. (1984), Simple linear-time algorithms to test chordality of graphs, test acyclicity of hypergraphs, and selectively reduce acyclic hypergraphs. SIAM J. on Computing, Vol.13, pp.566-579.

[19] Xue, J. (1994), Edge-maximal triangulated subgraphs and heuristics for the maximum clique problem. Networks, Vol.24, pp.109-120. 